\title{
Can Nonrenewable Resources Alleviate the Knife-edge Character of Endogenous Growth?
}

\section{Working paper}

\author{
Christian Groth* and Poul Schou** \\ Institute of Economics, University of Copenhagen
}

Corresponding author:

Christian Groth, Institute of Economics, University of Copenhagen,

Studiestraede 6, DK-1455

Copenhagen K, Denmark

tel: (+ 45) 353230 28, fax: $(+45) 35323000$

* e-mail: Chr.Groth@econ.ku.dk

** e-mail: Poul.Schou@econ.ku.dk

January 31, 2000 


\begin{abstract}
ES 2000

Can Nonrenewable Resources Alleviate the Knife-edge Character of Endogenous Growth?

by Christian Groth and Poul Schou

Corresponding author:

Christian Groth, Institute of Economics, University of Copenhagen, Studiestraede 6,

DK-1455 Copenhagen K., Denmark.

Chr.Groth@econ.ku.dk
\end{abstract}

Abstract: Can the knife-edge restriction on technology imposed by standard endogenous growth models be relaxed by allowing for nonrenewable resources entering the technology? To answer this question we examine whether stable endogenous growth is compatible with increasing returns to scale with respect to producible inputs when nonrenewable resources are a necessary input into the growth engine. In a one-sector optimal growth model, we find that the existence and stability of a steady state is compatible with a wide range of parameter values, including cases with increasing returns to capital and/or the natural resource. However, in all cases, population growth turns out to be necessary for stable growth in pr. capita consumption. Thus, under these circumstances, (strictly) endogenous stable growth is not possible, not even as a knife-edge case. But semi-endogenous growth is an attractive alternative allowing a rich set of determinants of long-run growth.

We thank Carl-Johan Dalgaard, University of Copenhagen, and Sjak Smulders, Tilburg University, for valuable comments on a first draft. 


\section{Introduction}

One of the weaknesses of endogenous growth models with balanced growth paths (e. g. Lucas 1988, Romer 1990, and Bond et al. 1996) is that exactly constant returns to scale with respect to the producible factors in the core sector of the economy is a necessary condition for steady positive per capita growth. Slightly increasing returns would lead to explosive growth, whereas slightly decreasing returns lead to growth petering out unless some exogenous factor (e.g., population) grows (cf. Solow, 1994). This is a serious challenge to the theory, as it implies lack of robustness to even slight parameter changes. The defining characteristic of these growth models (surveyed in Barro and Sala-i-Martin 1995) is that pr. capita consumption in the long run grows at a constant positive rate even if there is no exogenously given growth in any factor. We call this phenomenon (strictly) endogenous growth. Necessary for its existence is that the "growth engine" features the knife-edge property mentioned.

The purpose of this paper is to examine if more robust results can be obtained in a model where nonrenewable natural resources are a necessary input into the growth engine and where there is utility discounting and consumption smoothing. Intuition suggests that the strain on the economy imposed by the need to extract successively smaller amounts of the resource might offset the potentially explosive effects of increasing returns to scale with respect to the reproducible factor(s) of the model. Even if this intuition may be valid as far as the pure technical feasibility of growth is concerned, the problem of examining the restrictions implied by optimality and stability still remains.

Our approach to this problem is based on an extension of the basic onesector optimal growth model with non-renewable resources due to Stiglitz (1974) ${ }^{1}$. Stiglitz concentrates on the case of constant returns with respect to capital, labour, and the resource taken together. We extend the analysis, characterizing steady growth paths for a broader range of parameter values. In particular, in line with endogenous growth theory, we allow for a constant or increasing marginal prod-

\footnotetext{
${ }^{1}$ Dasgupta and Heal (1974, section 1) analyzed a rather similar model. For convenience, in the following we refer only to Stiglitz (1974).
} 
uct of capital as well as of the other two production factors, labour and natural resources.

Indeed, a constant marginal product with respect to (some kind of) capital is essential to most endogenous growth models (and all those that display balanced growth), such as the AK model. A non-decreasing marginal product may not seem so unrealistic when we recognize externalities or if we interpret "capital" broadly as a combination of physical capital, technical knowledge, and human capital. If a constant marginal product of capital is accepted, a slightly increasing marginal product should be considered as equally realistic. Of course, such conditions may not be consistent with a laissez faire market economy with perfect competition and even less with a socially optimal market economy. However, in the growth literature imperfect competition and/or tax and subsidy interventions à la Pigou are often viewed as institutions that bring about an allocation approximating the social optimum.

Whereas the Stiglitz (1974) model relies on exogenous technological progress to generate growth, a number of later papers like Robson (1980), Takayama (1980), Scholz and Ziemes (1996), Aghion \& Howitt (1998, pp. 163-64), and Schou (1999) have examined the implications of the presence of non-renewable resources in various endogenous growth models. Common for all these papers is, however, that the natural resource does not appear in the core sector (the growth engine) of the model (even not indirectly in the sense that resources are a necessary ingredient in the production of physical capital goods, which are then used in the growthcreating sector, e. g., a research sector). This is a crucial feature compared to the present model, and probably an unrealistic one. It seems unlikely that such sectors should be completely independent of physical capital and thus ultimately of the nonrenewable resource.

The present paper is related also to another model of Aghion \& Howitt (1998, pp. 162-63). They consider a one-sector AK-model with a nonrenewable resource added in Cobb-Douglas fashion. In that model, positive long-run growth is not possible. Our paper shows how this result may be reversed without violating stability when population growth and an explicit productive role for labour are 
introduced. By stressing the essential role of increasing returns and population growth for positive pr. capita growth (when there is no exogenous technology growth), our analysis has affinity with what Jones (1995) calls semi-endogenous growth as distinct from (strictly) endogenous growth. An early example of a semi-endogenous growth model is the famous learning-by-doing paper by Arrow (1962); another example is the modification of the Romer-1990 model suggested by Jones (1995). These "standard" semi-endogenous growth models (surveyed in Eicher \& Turnovsky 1999) differ from their (strictly) endogenous-growth relatives by having long-run growth rates that are independent of preference parameters. It turns out that when nonrenewable resources enter the growth engine, semiendogenous growth again assigns a role to preference parameters - hence also a role to fiscal policy - as a determinant of the long-run growth rate.

The organization of the paper is as follows. The next section presents the extended Stiglitz model and describes its steady state and transitional dynamics. In section 3, the necessary and sufficient conditions for existence and stability of a steady state in full generality are given followed by an examination of the conditions for stable steady states with positive pr. capita growth. Section 4 deals with a degenerate case arising when the output elasticity of the resource takes on a critical value. Section 5 accounts for some comparative dynamics properties. Conclusions are drawn in the final section.

\section{The Model}

Instantaneous utility of the representative infinitely-lived household is iso-elastic:

$$
\int_{0}^{\infty} \frac{c(t)^{1-\varepsilon}-1}{1-\varepsilon} N(t) e^{-\rho t} d t, \quad N(t)=N(0) e^{n t}, \varepsilon>0, \rho>n \geq 0, N(0)>0
$$

where $\mathrm{c}$ is per capita consumption, $\varepsilon$ is minus the elasticity of marginal utility, $\mathrm{N}$ is household size (interpreted as population size), $\rho$ is the rate of time preference, and $n$ is the rate of population growth. (From now on we will normally suppress the time argument.) To ensure that the nonrenewable resource is necessary for production, but does not a priori rule out sustainable consumption, we follow 
Stiglitz and assume an aggregate production function of Cobb-Douglas form:

$$
Y=A K^{\alpha} N^{\beta} R^{\gamma}, \quad A, \alpha, \beta, \gamma>0
$$

where $\mathrm{Y}$ is output of basic goods, $\mathrm{K}$ is the capital stock, and $\mathrm{R}$ is the flow of the nonrenewable resource (from now just called the resource). In contrast to Stiglitz (1974), we ignore exogenous technological progress. More importantly, we do not impose an upper bound on $\alpha, \beta$ or $\gamma$. At least, as mentioned in the introduction, a case can be made for larger values of these parameters than are assumed by Stiglitz who concentrates on the case $\alpha+\beta+\gamma=1$. A constant marginal product with respect to (some index of aggregate) capital (i.e., $\alpha=1$ ) is indeed standard and essential to most endogenous growth models. If $\mathrm{K}$ is interpreted as "broad capital" including technical knowledge and human capital, then the explicit role of $\mathrm{N}$ in the production function would represent the role of raw physical labour. If $\alpha=1$ is accepted, values slightly above unity should be allowed as well.

Empirical examinations of resources such as minerals and fossil fuels usually consider $\gamma$ to be considerably below unity, indeed, as low as 0.05 (according to Dasgupta \& Heal, 1979, p. 205). We want to note an alternative interpretation of the resource which makes the potential value range of $\gamma$ larger: $\mathrm{R}$ could be taken to represent pollution as an inevitable by-product of production, and possibly with a value of $\gamma$ considerably above 0.05 . In a situation with insufficient abatement possibilities, all pollution detracts from the quality $\mathrm{S}$ of the environment, considered to be a stock of "natural capital". If cumulated pollution keeps growing, then at a certain point the environmental stock is completely exhausted, and it will be impossible to pollute any more and hence to produce any further goods ${ }^{2}$. In a similar manner, Stokey (1998) presents a growth model where the modelling of pollution has some similarities with usual representations of a nonrenewable resource.

Whatever the interpretation of $\mathrm{R}$, output is used for consumption and for

\footnotetext{
${ }^{2}$ Though this interpretation is possible, in the following, to fix ideas we will refer to $\mathrm{R}$ as input of a natural resource.
} 
investment in capital goods, so that

$$
\dot{K}=Y-C-\delta K, \quad K(0)>0, \quad \delta \geq 0
$$

where $C \equiv c N$ is total consumption, and $\delta$ is the capital depreciation rate (disregarded in Stiglitz 1974). The resource stock $S$ diminishes with the flow of the resource:

$$
\dot{S}=-R, \quad S(0)>0 .
$$

The social planner wants to maximize 2.1 subject to $2.2-4$ and to the conditions

$$
c>0, R \geq 0
$$

for the control variables, and

$$
K \geq 0, S \geq 0
$$

for the state variables.

The current-value Hamiltonian for this problem is:

$$
H=\frac{c^{1-\varepsilon}}{1-\varepsilon} N+\mu_{K}\left(A K^{\alpha} N^{\beta} R^{\gamma}-c N-\delta K\right)-\mu_{S} R
$$

Necessary conditions for an interior solution are given by the following first-order and transversality conditions:

$$
\begin{gathered}
c^{-\varepsilon}=\mu_{K} \\
\mu_{K} \gamma \frac{Y}{R}=\mu_{S} \\
\frac{\dot{\mu}_{K}}{\mu_{K}}=\rho-\alpha \frac{Y}{K}+\delta \\
\frac{\dot{\mu}_{S}}{\mu_{S}}=\rho \\
\lim _{t \rightarrow \infty} e^{-\rho t} \mu_{K} K=0 \\
\lim _{t \rightarrow \infty} e^{-\rho t} \mu_{S} S=0
\end{gathered}
$$

where $\mu_{K}$ and $\mu_{S}$ are the co-state variables associated with physical capital and 
the resource stock, respectively.

Differentiating the production function 2.2 with respect to time, we get

$$
g_{Y}=\alpha g_{K}+\beta n+\gamma g_{R}
$$

(where $g_{i}$ denotes the growth rate of variable $i$ ). Differentiating 2.8 with respect to time and combining with 2.10 gives the Keynes-Ramsey rule:

$$
g_{c}=\frac{Y_{K}-\delta-\rho}{\varepsilon}
$$

where $Y_{K}$ is the marginal product of capital. Similarly, differentiating 2.9 with respect to time and combining with 2.10 and 2.11 gives the Hotelling rule for optimal extraction of a non-renewable resource:

$$
\frac{\dot{Y}_{R}}{Y_{R}}=Y_{K}-\delta
$$

\subsection{Steady State}

We define a steady state as a path along which $\mathrm{Y}, \mathrm{R}, \mathrm{C}, \mathrm{K}$, and $\mathrm{S}$ are positive and change with constant, though not necessarily positive rates. Along such a path, output, capital and (total) consumption must have the same growth rate (otherwise, 2.3 could not hold for all $\mathrm{t}$ ). This common growth rate we call $\mathrm{g}$. Combining 2.15 and 2.16 and making use of the Cobb-Douglas specification, we can derive the following steady-state condition:

$$
g_{R}=(1-\varepsilon) g+\varepsilon n-\rho
$$

Now, let

$$
D \equiv 1-\alpha+(\varepsilon-1) \gamma
$$

Observe that $\mathrm{D}$ does not depend on $\rho, \beta, n$, and $\delta$. Assume

$$
D \neq 0
$$


Then, 2.17 together with 2.14 (with $g_{Y}=g_{K}=g$ ) results in the following steadystate growth rates, presupposing that a steady state exists ${ }^{3}$ :

$$
\begin{aligned}
g & =\frac{-\gamma \rho+(\beta+\gamma \varepsilon) n}{D} \\
g_{R} & =\frac{(\alpha-1) \rho+(\varepsilon(1-\alpha-\beta)+\beta) n}{D} \\
g_{c} & =g-n=\frac{-\gamma \rho+(\alpha+\beta+\gamma-1) n}{D} .
\end{aligned}
$$

Define the following ratios:

$$
x=\frac{C}{K}, \quad z=\frac{Y}{K}, \quad u=\frac{R}{S} .
$$

$\mathrm{x}, \mathrm{z}$ and $\mathrm{u}$ are the consumption-capital ratio, output-capital ratio and resource extraction rate, respectively. These ratios will be constant in the steady state. Indeed, for $x$ and $z$ this follows from 2.3 and it applies to $u$ as well, since, by 2.4 , $\mathrm{u}=-g_{S}$, which is, by definition, constant in a steady state. Using 2.15, 2.20, 2.3, 2.18 and 2.19, the steady-state values (denoted by an asterisk) of these three variables are found:

$$
\begin{gathered}
x^{*}=\frac{(1-\alpha)(1-\gamma) \rho+(\varepsilon(\beta-(1-\alpha)(1-\gamma))-\alpha \beta) n}{\alpha D}+\frac{1-\alpha}{\alpha} \delta \\
z^{*}=\frac{(1-\alpha-\gamma) \rho+(\alpha+\beta+\gamma-1) \varepsilon n}{\alpha D}+\frac{\delta}{\alpha} \\
u^{*}=-g_{R}=\frac{(1-\alpha) \rho-(\varepsilon(1-\alpha-\beta)+\beta) n}{D} .
\end{gathered}
$$

As we have defined a steady state, it only exists if these three values are positive. It is because of the endogenous growth perspective of this paper that our definition of a steady state disregards the conceivable asymptotic stationary states where $x, z$, and/or $u$ approach zero, cf., e.g., Dasgupta and Heal (1974).

\footnotetext{
${ }^{3}$ When $D=0$, there will be either no or, as a knife-edge case, infinitely many steadystate growth rates $g$ and $g_{R}$ fulfilling the necessary conditions for optimality. To avoid these unimportant complexities, we assume in the remainder of this paper that A1 holds.
} 
The expressions 2.18-20 and 2.22-24 are straightforward generalizations of corresponding expressions in Stiglitz (1974). The interesting question is how the larger range allowed for the parameters affects the existence and character of steady states. However, we defer the examination of this question until after a presentation of the transitional dynamics.

\subsection{Transitional Dynamics}

It follows from 2.21, 2.3 and 2.4 that

$$
\begin{gathered}
g_{K}=z-x-\delta \\
g_{x}=g_{C}-z+x+\delta \\
g_{z}=g_{Y}-z+x+\delta \\
g_{u}=g_{R}+u .
\end{gathered}
$$

Using the Cobb-Douglas specification, the Hotelling rule 2.16 can be rewritten as

$$
g_{R}=g_{Y}-\alpha z+\delta
$$

In the same way the Keynes-Ramsey rule 2.15 together with 2.26 implies

$$
g_{x}=x+\left(\frac{\alpha}{\varepsilon}-1\right) z-\frac{\delta+\rho}{\varepsilon}+n+\delta .
$$

Inserting 2.25 and 2.29 into 2.14 yields, for $\gamma \neq 1^{4}$,

$$
g_{Y}=-\frac{\alpha}{1-\gamma} x+\alpha z+\frac{\beta n+(\gamma-\alpha) \delta}{1-\gamma} .
$$

Using this expression in 2.27 , we find

$$
g_{z}=\frac{1-\alpha-\gamma}{1-\gamma} x+(\alpha-1) z+\frac{\beta n+(1-\alpha) \delta}{1-\gamma} .
$$

The growth of $\mathrm{u}$ is given by $2.28,2.29$ and 2.31 :

$$
g_{u}=\frac{-\alpha}{1-\gamma} x+\frac{\beta n+(1-\alpha) \delta}{1-\gamma}+u .
$$

\footnotetext{
${ }^{4}$ The case $\gamma=1$ is studied in section 4 .
} 
The dynamical system for $x, z$ and $u$ is completely described by 2.30, 2.32 and 2.33. The system is decomposeable. 2.30 and 2.32 constitute a dynamical subsystem for $x$ and $z$ alone. In steady state, $g_{x}=g_{z}=0$ (otherwise, 2.3 could not hold for all $t$ ). We can form the steady-state Jacobian:

$$
J=\left[\begin{array}{ll}
\frac{\partial \dot{x}}{\partial x} & \frac{\partial \dot{x}}{\partial z} \\
\frac{\partial \dot{z}}{\partial x} & \frac{\partial \tilde{z}}{\partial z}
\end{array}\right]=\left[\begin{array}{cc}
x^{*} & \left(\frac{\alpha}{\varepsilon}-1\right) x^{*} \\
\frac{1-\alpha-\gamma}{1-\gamma} z^{*} & (\alpha-1) z^{*}
\end{array}\right]
$$

where a star denotes a steady-state value. The determinant is

$$
z^{*} x^{*} \alpha \frac{1-\alpha+(\varepsilon-1) \gamma}{(\gamma-1) \varepsilon} \equiv z^{*} x^{*} \alpha \frac{D}{(\gamma-1) \varepsilon}
$$

which is negative (hence, implying eigen values of opposite sign) if and only if

$$
\text { either }(D>0 \text { and } \gamma<1) \quad \text { or } \quad(D<0 \text { and } \gamma>1) \text {. }
$$

In the case of constant returns to scale $(\alpha+\beta+\gamma=1)$, analyzed by Stiglitz (1974), the condition $(D>0$ and $\gamma<1)$ is automatically satisfied.

In figure 2.1, a phase diagram is drawn for a particular constellation of the parameters satisfying inter alia $0<\gamma<1 \approx \alpha<\varepsilon$ so that 2.34 applies.

Along the saddle path, we have approximately

$$
\begin{aligned}
\dot{z} & =\lambda\left(z^{*}-z\right), \\
x & =f(z)
\end{aligned}
$$

where the constant $\lambda$ is minus the negative eigen value of $J$, and $f$ is a linear function that can be determined by standard methods.

Using this fact, we can construct a phase diagram in $\mathrm{z}$ and $\mathrm{u}$ (figure 2.2), using 2.35 and 2.33 , where $\mathrm{x}$ is replaced by $\mathrm{f}(\mathrm{z})$. The determinant of the Jacobian of this system is $-\lambda u^{*}<0$.

Even though z, x, and u are all "jump variables", by substituting uS for R in the production function 2.2 we get

$$
z=A K^{\alpha-1} N^{\beta} u^{\gamma} S^{\gamma}
$$




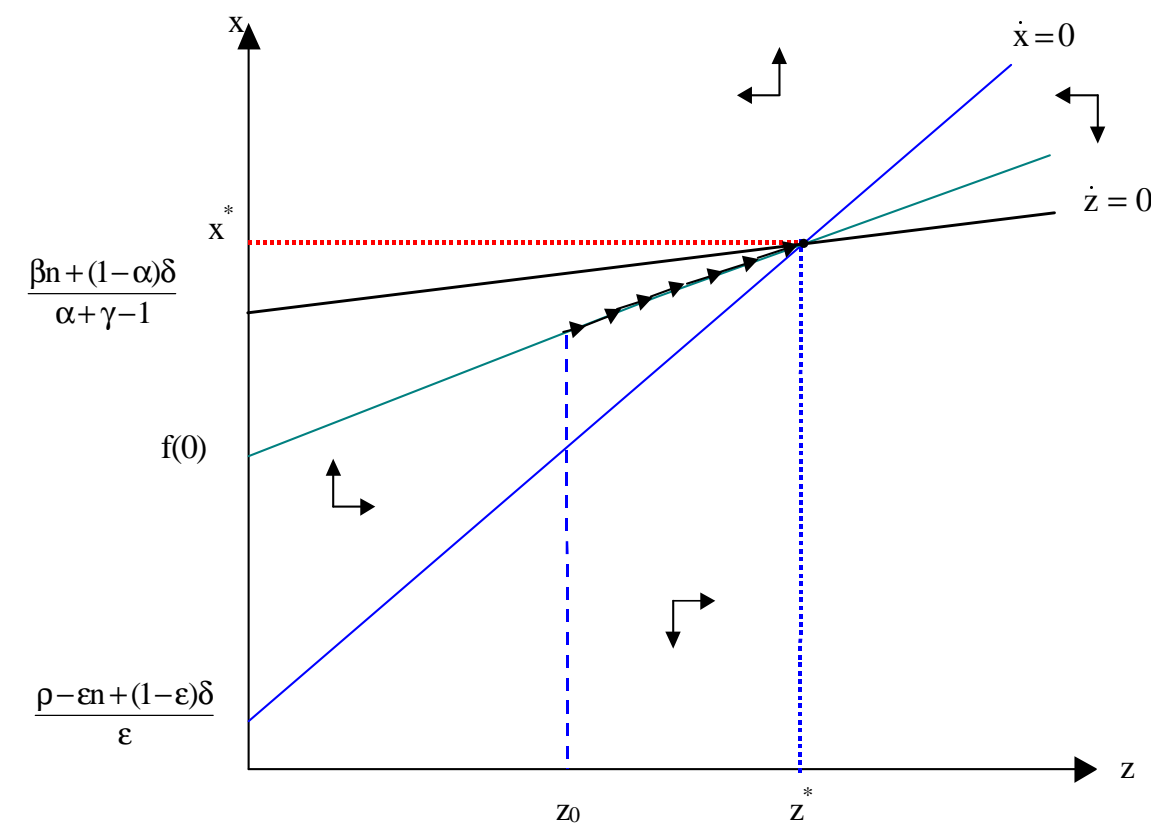

Figure 2.1: Dynamics of $z$ and $x$ (when $\gamma<1$, slightly above 1 , and $\varepsilon>\alpha$ ).

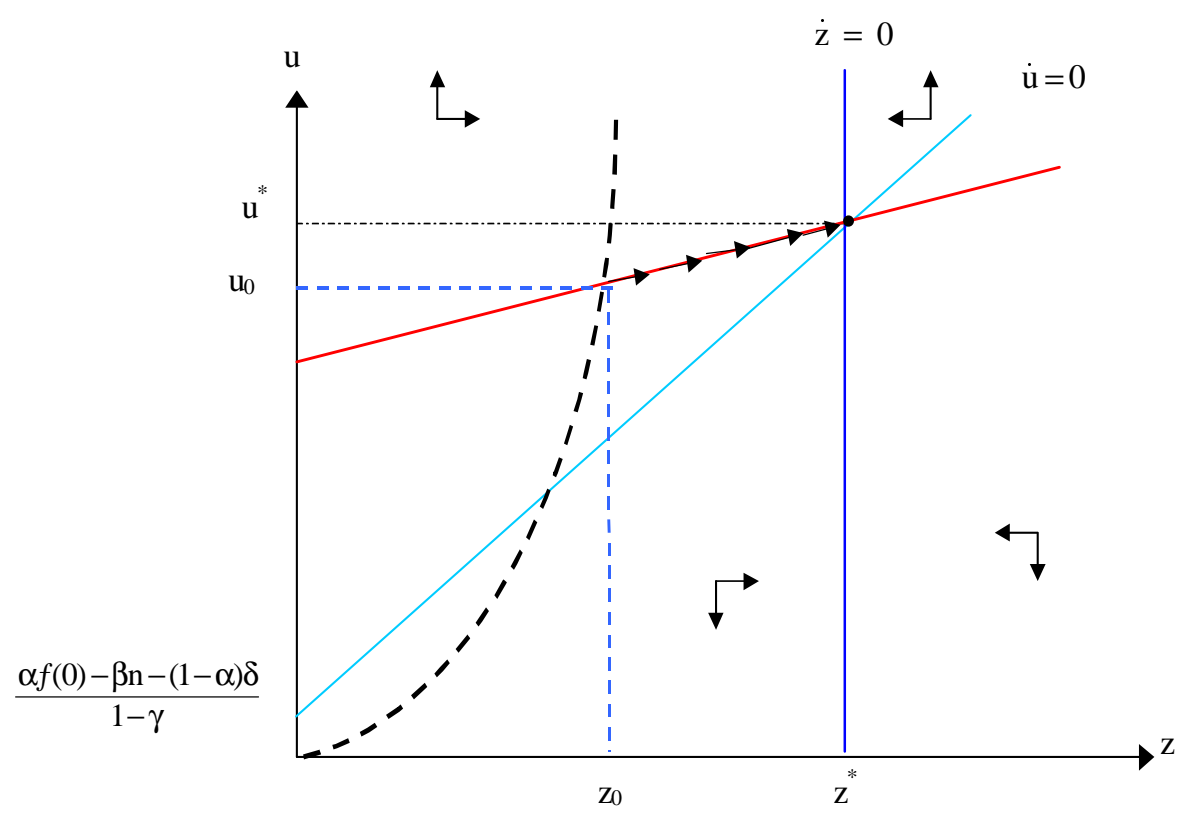

Figure 2.2: Dynamics of $z$ and $u$. 
showing that there is a bound between the values of $\mathrm{z}$ and $\mathrm{u}$ which must hold at any t; thus, initially, $u_{0}=\left(A^{-1} K_{0}^{1-\alpha} L_{0}^{-\beta} S_{0}^{-\gamma} z_{0}\right)^{\frac{1}{\gamma}}$, cf. the dotted curve in figure 2.2. The intersection between this curve and the saddle-path determines uniquely the initial values of $\mathrm{z}$ and $\mathrm{u}, \mathrm{z}_{0}$ and $\mathrm{u}_{0}$, required for convergence to the steady state. The unique intersection between the line $z=z_{0}$ and the saddle path in figure 2.1 gives the required $x_{0}$. Then, presupposing convergence, the movement of the economy over time is along the saddle-paths of figures 2.1 and 2.2. (Again, this analysis is just an outright extension of the corresponding stability analysis in Stiglitz (1974). The slopes of the $\dot{x}=0$ and the $\dot{z}=0$ lines in figure 2.1 may be either positive or negative, depending on the parameters; in case $\gamma<1$, if $\alpha$ is slightly above 1 , then the $\dot{z}=0$ line has positive slope and so has the $\dot{x}=0$ line if there is to be stability.)

\section{The Existence and Character of Steady States}

We want to characterize the parameter set ensuring the existence and stability of a steady state fulfilling the stated first-order and transversality conditions. In particular, our emphasis will be on steady states with an increasing rate of pr. capita consumption.

Proposition 3.1. Given the model 2.1-2.6, assume A1. A steady state (fulfilling the first-order and transversality conditions above) exists if and only if the righthand sides of 2.22, 2.23 and 2.24 are positive. If, in addition, $\gamma \neq 1$, then ${ }^{5}$ :

a) this steady state is saddle point stable if and only if 2.34 holds and it is totally unstable if 2.34 does not hold;

b) a stable steady state has increasing pr. capita consumption if and only if the right-hand side of 2.20 is positive;

c) when $\gamma<1$, a stable steady state has non-decreasing pr. capita consumption if and only if $\alpha+\beta>1$ and $n \geq \gamma \rho /(\alpha+\beta+\gamma-1)$.

Proof: See appendix.

\footnotetext{
${ }^{5}$ As to the case $\gamma=1$, see section 4 .
} 
The proposition implies that there exists a stable steady state also outside the range of parameter values examined in Stiglitz $(1974)^{6}$. Indeed, as we shall see below, existence is compatible with values of $\alpha$ (and/or $\gamma$ ) above unity, so that there can be increasing returns to scale with respect to capital (as well as to the resource). Statement a) also has the implication that a multiplicity of convergent paths never arises; either a steady state is saddle point stable or it is totally unstable ${ }^{7}$. Further, as will become clear in the sequel, the set of parameter values implying a stable steady state with growing pr. capita consumption is a non-empty open set. As to statement c) of the proposition, the implication is that, in the realistic case $\gamma<1$, increasing returns to scale with respect to capital and labour taken together as well as enough population growth are required to offset the effects of the decreasing input of the natural resource if a stable steady state is to exist ${ }^{8}$.

It is not easy to state, in full generality, the various parameter conditions in a more informative and concise way than done in proposition 1. We can, however, do this for three particular benchmark cases: The case of no population growth $(n$ $=0)$, the case of constant returns to capital $(\alpha=1)$, and the case of logarithmic utility $(\varepsilon=1)$.

\section{Case 1: No Population Growth}

Proposition 3.2. Assume $A 1$ and $n=0$. Then there can be no steady state (as here defined) with $g_{c}=0$. There exists a steady state with $g_{c}>0$ if and only if

$$
\begin{aligned}
& D<0 \\
& \alpha>1 \\
& \gamma<1
\end{aligned}
$$

\footnotetext{
${ }^{6}$ It can be shown that Arrow's sufficient conditions for optimality are fulfilled if and only if $\alpha+\gamma \leq 1$. We conjecture that even outside this range (at least as long as $D>0$ ), a steady state growth path fulfilling the necessary first-order and transversality conditions above is indeed optimal.

${ }^{7}$ This holds even if $\gamma=1$ is allowed, since $\gamma=1$ automatically ensures "saddle point stability", properly defined, cf. section 4.

${ }^{8}$ Remember that due to the endogenous growth perspective of the present paper, we disregard the possible asymptotic steady states where $x, z$, and/or $u$ approach zero.
} 


$$
\rho>\frac{-D \delta}{1-\gamma}
$$

A steady state with $g_{c}>0$ is completely unstable.

Proof: See appendix.

That $g_{c}>0$ requires $\alpha>1$ is seen from the Keynes-Ramsey rule and the fact that otherwise, the marginal product of capital would tend to zero as capital accumulates and resource use diminishes. The proposition sharpens a remark made by Rebelo (1991, p. 519) in that sufficiency requires not only $\alpha \geq 1$ (as claimed by Rebelo), but $\alpha>1$ and $\gamma<1$ (because a not too large productive importance of the resource is required in order that capital accumulation can offset the effect of the diminishing resource use $)^{9}$. In any case, due to its lack of stability, this steady state is not very appealing.

But an interesting implication arises when we compare proposition 3.2 with the fact that, even with no population growth, capital accumulation can, from a purely technical point of view, support any positive constant consumption growth rate as soon as $\alpha>1$ and $\gamma<1$ (in the case $\delta=0$ ). This follows easily by studying technically efficient paths (defined as technically feasible paths satisfying the Hotelling rule, but not necessarily the Keynes-Ramsey rule), using the method of Stiglitz $(1974 \text {, section } 2)^{10}$. This is also what intuition suggests, namely, first that the strain on the economy imposed by the need to extract successively smaller amounts of the resource can be offset by increasing returns to scale with respect to capital, and second that on the other hand the potentially explosive effects of this type of increasing returns may be exactly counterbalanced by the dimishing resource use. Now, proposition 3.2 not only tells us that a desire for consumption smoothing combined with utility discounting restricts the feasibility of steady states with $\mathrm{g}_{c}>0$ when $n=0$, but also that it completely excludes stability of such paths.

\footnotetext{
${ }^{9}$ Further, Rebelo's remark is concerned only with feasibility and does not relate to optimality and stability.

${ }^{10}$ Section 2 of the Stiglitz paper studies such technically efficient growth paths. Mostly concentrating on the CRS case, $\alpha+\beta+\gamma=1$, Stiglitz does not point at the conclusion mentioned. Optimal growth in the CRS case is the topic of his section 3.
} 
This has implications for the ongoing discussion on endogenous growth. We have defined (strictly) endogenous growth to occur if pr. capita consumption in the long run grows at a positive constant rate even if there is no exogenously given growth in any variable. Likewise, weakly endogenous or semi-endogenous growth (as introduced by Groth (1992) and Jones (1995)) is said to occur if pr. capita consumption in the long run grows at a positive rate without any exogenously given technology growth. With these definitions, we have the following:

Corollary. In a Cobb-Douglas one-sector optimal growth model with nonrenewable natural resources, a stable steady state with endogenous growth does not exist, not even as a knife-edge case. ${ }^{11}$

The model, however, may display stable semi-endogenous growth. As proposition 3.1 indicates and as we will explore in more detail below, there may be stable positive pr. capita growth, but a necessary condition for this is that there is enough population growth. It is worth mentioning that in contrast to standard semi-endogenous growth models (surveyed in Eicher and Turnovsky 1999), we find that when nonrenewable resources enter the growth engine, semiendogenous growth features an interesting property similar to that of strictly endogenous growth. This is the property that the long-run growth rate depends on technology as well as preference parameters, thereby allowing even a direct role to fiscal policy.

\section{Case 2: Constant Returns to Capital $(\alpha=1)$}

Here we consider an AK model augmented with an explicit role for both the labour force and natural resources in production. In this case the $\dot{z}=0$ curve in figure 1 is horizontal. The growth rates 2.19 and 2.20 reduce to

$$
\begin{aligned}
g_{R} & =\frac{-\beta n}{\gamma} \\
g_{c} & =\frac{-\gamma \rho+(\beta+\gamma) n}{(\varepsilon-1) \gamma},
\end{aligned}
$$

\footnotetext{
${ }^{11}$ Obviously, this conclusion also holds if, instead of the classical utilitarian criterion in 2.1, based on "total utility", the "average utility"-criterion had been used (eliminate the factor $N(t)$ in 2.1) since, when $n=0$, the two criteria are the same.
} 
and assumption A1 implies $\varepsilon \neq 1$. We have the following

Proposition 3.3. Assume $A 1$ and $\alpha=1$. Then there exists a steady state with $g_{c}>0$ if and only if $n>0$ and

$$
\begin{aligned}
\text { either } \varepsilon & >1 \wedge \gamma<\frac{\beta n}{\rho-n} \\
\text { or } \varepsilon & <1 \wedge \gamma>\frac{\beta n}{\rho-n} .
\end{aligned}
$$

When additionally $\gamma \neq 1$, the steady state is stable if and only if

$$
\text { either } \varepsilon>1 \wedge \gamma<1 \text { or } \varepsilon<1 \wedge \gamma>1 \text {. }
$$

Proof: See appendix.

When $\alpha=1$, a low willingness to intertemporal substitution (high $\varepsilon$ ) requires a counterweight in the form of a small output elasticity with respect to the resource. Population growth still can ensure stable pr. capita consumption growth in the long run. This contrasts with the model without resources $(\gamma=0)$, where a situation with $\alpha=1, \beta>0$ and population growth is not compatible with a steady state, but implies explosive growth. In the present model, population growth is not only compatible, but necessary for positive stable growth. One of the models examined in Aghion \& Howitt (1998, pp. 162-63) is an AK model with a non-renewable resource. In that model, however, labour does not appear in the production function. Because of this, in that model positive long-run growth is not possible ${ }^{12}$.

A different perspective shows up if one accepts the Solow view that there is probably an irreducibly exogenous element in the development of new technology in society, at least exogenous to the economy (Solow 1994, p. 51). Following this

\footnotetext{
${ }^{12}$ Aghion \& Howitt carry on with another model based on what they call a Schumpeterian approach to non-renewable resources (Aghion \& Howitt 1998, pp. 163-64). Here the economy has two sectors, a manufacturing sector and a R \& D sector, both with constant returns to scale to producible inputs. The authors find that now growth is possible (without population growth) and they ascribe this to the Schumpeterian approach as distinct from the AK approach. In our view, the key is rather that the "growth engine" (the R \& D sector) is modelled as not depending on the resource (not even indirectly since the R \& D sector does not use capital).
} 
strand, with a slight modification of the model, the important parameter $n$ could be reinterpreted as representing this exogenous element.

Case 3: Logarithmic Utility $(\varepsilon=1)$.

In this case, from $2.24, u^{*}=\rho-n$ (a nice simple expression also noted by Stiglitz).

Proposition 3.4. Assume $A 1$ and $\varepsilon=1$. Then there exists a steady state with $g_{c}>0$ if and only if

$$
\begin{aligned}
\text { either } & \alpha<1 \wedge \gamma<\frac{\alpha+\beta-1}{\rho-n} n \wedge n>0 \\
\text { or } & \alpha>1 \wedge \frac{\alpha+\beta-1}{\rho-n} n<\gamma<1+\frac{\beta n-(\alpha-1) \delta}{\rho-n} .
\end{aligned}
$$

When additionally $\gamma \neq 1$, the steady state is stable if and only if

$$
\text { either } \quad \alpha<1 \wedge \gamma<1 \quad \text { or } \quad \alpha>1 \wedge \gamma>1 \wedge n>0 \text {. }
$$

Proof: See appendix.

The proposition implies that a necessary condition for steady positive pr. capita growth when $\varepsilon=1$ is $\alpha+\beta>1$. At the same time, there is a certain relation between the sizes of $\alpha$ and $\gamma$. When the output elasticity with respect to man-made capital, $\alpha$, is below a critical level, equal to 1 , then also the output elasticity with respect to the resource, $\gamma$, must have an upper bound. Conversely, when $\alpha$ is large (above 1), $\gamma$ must be not too small. Capital and the resource have counter-acting influences on the possibilities for steady growth, so their importance in production cannot differ too much. On the other hand, when $\alpha$ is above 1 , then $\gamma$ must also not be too large because that would make it impossible for capital accumulation to offset the diminishing resource input. It is also seen that if one of either $\alpha$ or $\gamma$ is above 1 , then stability of a steady state with $g_{c}>0$ requires that both $\alpha$ and $\gamma$ are above 1 . 


\section{The Degenerate Case $\gamma=1$}

When $\gamma=1$, the formulas up to 2.30 are still valid, i.e., the steady state properties are not qualitatively affected. But the transitional dynamics is changed radically. Indeed, calculating the equivalents of 2.31 and 2.30 shows that there will be no transitional dynamics, given A1 (implying $\alpha \neq \varepsilon$, in this case). The system degenerates into a recursive structure: $\mathrm{x}$ immediately jumps to its steady-state value, $(\beta n+(1-\alpha) \delta) / \alpha$, independently of the rest of the system, implying that in turn $\mathrm{z}$ and then $\mathrm{u}$ take on their steady-state values. (It is convenient to extend the notion of saddle point-stability to cover even this case.) Further, in this situation 2.20 shows that the existence of an optimal steady state with growing pr. capita consumption again requires a growing population ${ }^{13}$.

\section{Comparison of Steady States}

Assuming that the right-hand sides of 2.22-24 are positive so that a steady state exists, we can examine the consequences for the constant growth rates 2.19 and 2.20 of varying the various parameters of the model. First, we note that neither the productivity parameter $\mathrm{A}$ nor the depreciation rate $\delta$ affects the steady-state growth rate at all, in contrast to the usual one-sector endogenous growth model (the AK model); this is true even when $\alpha=1$. (But $\delta$ enters the conditions for the existence of a steady state, cf. 2.22-24.) It is interesting that all the remaining parameters of the model affect the rate of pr. capita growth. Hence, there is a potential for tax and subsidy policies to influence not only the "level" along which growth occurs, but also the long-run growth rate. This is in contrast to standard semi-endogenous growth models where policy has only level effects (cf. Jones 1995).

Regarding the sign of the effect on growth of a change in a parameter, the results depend crucially on the sign of the denominator $\mathrm{D}$. We distinguish between

\footnotetext{
${ }^{13}$ Suppose $\gamma=1$, and $n=0$. Then, by $2.20, \mathrm{~g}_{c}>0 \Rightarrow D<0 \Rightarrow \alpha>\varepsilon \Rightarrow \alpha>1$ since, by $2.24, u *=(1-\alpha) \rho /(\varepsilon-\alpha)>0$. But $\alpha>1$ contradicts $x>0$, by 2.22 .
} 
the standard case ${ }^{14}(\mathrm{D}>0)$ and the "paradoxical" case $(\mathrm{D}<0)$.

\subsection{The Standard Case $(\mathrm{D}>0)$}

In this case, using (2.20), consumption pr. capita is seen to grow in the steady state if and only if

$$
(\alpha+\beta+\gamma-1) n>\gamma \rho
$$

which means that positive population growth and increasing returns to scale for capital and labour taken together in the production sector $(\alpha+\beta>1)$ are both necessary conditions for a steady state with growing pr. capita consumption (the last condition follows because $\gamma \rho>\gamma n$ ). More growth in population (often considered detrimental to pr. capita growth) affects pr. capita consumption growth positively, as long as there are increasing returns to scale. This is the net result of three different effects: There is a direct positive effect on output growth because a growing population means a growing labour force, magnifying the effects of increasing returns to scale. In addition, there is an indirect production effect because population growth also affects resource extraction (as seen from 2.19), though the sign of this effect is ambiguous. Thirdly, given the growth rate of total production, a larger population growth rate naturally means a smaller growth in pr. capita consumption possibilities.

2.20 also shows the familiar effect from Stiglitz (1974) that a rise in impatience (larger $\rho$ ) will affect the consumption growth rate negatively; and according to 2.24 this will be accompanied by an increased resource extraction rate, causing the resource to diminish faster over time. A larger value of $\rho$ will also cause a smaller consumption growth rate when $\alpha>1$; interestingly, however, in this case the extraction rate will fall. The reason must be that when the output elasticity of capital is high, smaller capital investment is required so that a high initial consumption level can be obtained with lower output early on, hence lower extraction of the resource early on.

The effect of a rise in $\alpha, \beta$ or $\gamma$ (a permanent technology shock) on the pr. capita consumption growth rate will be:

\footnotetext{
${ }^{14}$ Called so, because it is implied by the standard assumption, $\alpha+\beta+\gamma=1$.
} 


$$
\begin{aligned}
& \frac{\partial g_{c}}{\partial \beta}=\frac{n}{D} \geq 0 \\
& \frac{\partial g_{c}}{\partial \gamma}=\frac{g_{R}}{D}<0, \\
& \frac{\partial g_{c}}{\partial \alpha}=\frac{g}{D} .
\end{aligned}
$$

Not surprisingly, a rise in $\beta$, the output elasticity of labour, will raise the growth rate of consumption, whereas a rise in $\gamma$ will lower it ( $\gamma$ measures the importance of the resource in production; the need to save increasingly on the resource is reflected in the negative effect of this parameter on growth). The effect of $\alpha$ on consumption growth depends on the sign of $g$, i.e., the growth rate of total production: When output is growing then also capital is growing and an increase in the output elasticity of capital, $\alpha$, will increase growth further; similarly, when total output contracts, a higher value of $\alpha$ implies a decrease in the already negative pr. capita growth rate because the capital stock is falling, and a larger importance of capital for production will speed up the contraction.

The sign of the effect on the extraction rate of a rise in $\alpha, \beta$, or $\gamma$, depends on the desire for consumption smoothing as measured by $\varepsilon$. By 2.24, we have

$$
-\frac{\partial g_{R}}{\partial i}=\frac{\partial u^{*}}{\partial i}=(\varepsilon-1) \frac{\partial g_{c}}{\partial i}, \quad i=\alpha, \beta, \gamma
$$

where $\frac{\partial g_{c}}{\partial i}$ is given in 5.2-5.4. When people are very concerned with smoothing consumption over time $(\varepsilon>1)$, the sign of the effect on $u^{*}$ will echo that of the effect on consumption growth. The resource extraction rate will adjust to counteract the effect on the consumption level (caused by changed capital investment when growth changes) to spread out the potential gains or losses from a permanent technology shock more evenly over time. Conversely, when the desire for consumption smoothing is small, resource extraction is not prevented from adjusting in a complementary way to the change in the future marginal productivity of the resource caused by capital accumulation.

The effect of a change in $\varepsilon$ itself is

$$
\frac{\partial g_{c}}{\partial \varepsilon}=\frac{-\gamma g_{c}}{D}
$$




$$
-\frac{\partial g_{R}}{\partial \varepsilon}=\frac{\partial u^{*}}{\partial \varepsilon}=\frac{(1-\alpha) g_{c}}{D}
$$

Naturally, a larger $\varepsilon$ (preference for consumption smoothing) will lead to a numerically smaller consumption growth rate. Indeed, from 2.20 , if $\varepsilon \rightarrow \infty$, then $g_{c} \rightarrow 0$. The sign of the effect on the extraction rate is the same as that of $(1-\alpha) g_{c}$.

\subsection{The Paradoxical Case $(\mathrm{D}<0)$}

The denominator $D$ is negative if and only if $\alpha+\gamma>1+\varepsilon \gamma$, i.e., the elasticity of scale with respect to capital and the resource taken together must be considerably above 1 . When $D$ is negative, all the formulas from the standard case are valid, but the signs of the effects of parameter changes are reversed. Indeed, more impatience results in higher pr. capita consumption growth, and higher population growth implies lower pr. capita consumption growth, a rise in $\alpha$ will reduce consumption growth when this is already positive, a higher elasticity of marginal utility leads to larger differences in the consumption level over time, etc. All these results are highly counterintuitive, but they are nevertheless compatible with the existence of a stable steady state that exhibits positive pr. capita growth and satisfies the firstorder and transversality conditions 2.8-2.13. It cannot be excluded, however, that in the case $D<0$ these conditions do not signify optimality. However that may be, the realism of the parameter values generating this situation is questionable (as we have seen, stability in the case $D<0$ requires $\gamma \geq 1$ !).

\section{Conclusion}

We have studied a Cobb-Douglas one-sector optimal growth model with nonrenewable resources (and no exogenous technological progress). A stable steady state exists for a larger range of values than examined in Stiglitz (1974), and this larger range also allows for some new comparative dynamics results (even in the standard case $\mathrm{D}>0$ ). Indeed, existence and stability of a steady state was shown to be compatible with increasing returns to scale with respect to the reproducible factor capital and/or the natural resource. A high output elasticity of capital need not lead to explosive growth paths because the need to save on the 
natural resource has a counter-balancing effect. Hence, the knife-edge character of usual endogenous growth models without nonrenewable resources is not present. However, population growth is a necessary condition for a stable optimal steady state with positive pr. capita growth. Therefore, the extended Stiglitz optimal growth model is not consistent with endogenous growth in the strict sense of the word, i.e., the model cannot generate positive and stable pr. capita growth without relying on population growth.

The conclusion is that the challenge of generating a generic model with strictly endogenous growth cannot be met by simply including nonrenewable natural resources. On the contrary, in this setting the phenomenon of stable endogenous growth in the strict sense does not exist even as a knife-edge possibility! Hence, to the extent that the presence of such indispensable natural resources in a macro production function is considered realistic, the analysis has identified a new problem for the concept of strictly endogenous growth: It seems not possible to have stable, strictly endogenous growth at all when non-renewable resources enter the growth engine. This also applies, then, to market economies if they are considered to be replicae, through tax and subsidy interventions, of social optima. Therefore, the perspective suggested by the analysis is semi-endogenous growth.

It is a possibility (but we think an unlikely one) that this result could change in a multi-sector model where the reproducible asset(s) generating growth would depend on the resource (possibly indirectly). (The multi-sector endogenous growth models with non-renewable resources mentioned in the introduction all share the feature that the growth engine is not dependent on the resource). This problem is left for future research.

A slightly different perspective shows up if one accepts the Solow view that there is probably an irreducibly exogenous element in the development of new technology in society, at least exogenous to the economy (Solow 1994, p. 51). Following this strand, with a modification of the model, the important parameter $n$ could be reinterpreted as representing this exogenous element and we could proceed with a kind of quasi-endogenous growth analysis based on this notion. Interestingly, when nonrenewable resources enter the growth engine, both semi- 
endogenous growth and quasi-endogenous growth feature the property of standard (strictly) endogenous growth that the long-run growth rate depends on technology as well as preference parameters, thus allowing even a direct role to fiscal policy.

\section{Appendix}

\subsection{Stability properties of the three-dimensional system when $\gamma \neq 1$.}

The Jacobian of the dynamical system for $\mathrm{x}, \mathrm{z}$ and $\mathrm{u}$, evaluated in the steady state, when $\gamma \neq 1$, is:

$$
J=\left[\begin{array}{lll}
\frac{\partial \dot{x}}{\partial x} & \frac{\partial \dot{x}}{\partial z} & \frac{\partial \dot{x}}{\partial u} \\
\frac{\partial z}{\partial x} & \frac{\partial \dot{z}}{\partial z} & \frac{\partial \dot{z}}{\partial u} \\
\frac{\partial u}{\partial x} & \frac{\partial u}{\partial z} & \frac{\partial u}{\partial u}
\end{array}\right]=\left[\begin{array}{ccc}
x^{*} & \left(\frac{\alpha}{\varepsilon}-1\right) x^{*} & 0 \\
\frac{1-\alpha-\gamma}{1-\gamma} z^{*} & (\alpha-1) z^{*} & 0 \\
\frac{-\alpha}{1-\gamma} u^{*} & 0 & u^{*}
\end{array}\right]
$$

The trace is

$$
x^{*}+(\alpha-1) z^{*}+u^{*}=2 u^{*}>0
$$

where we have used $2.25,2.29$, and 2.28 . The determinant is

$$
x^{*} z^{*} u^{*} \alpha \frac{1-\alpha+(\varepsilon-1) \gamma}{(\gamma-1) \varepsilon}=x^{*} z^{*} u^{*} \alpha \frac{D}{(\gamma-1) \varepsilon}
$$

which is negative if and only if 2.34 holds. Hence, 2.34 implies one eigenvalue with negative real value and two eigenvalues with positive real value, which is just what is required for saddle-point-stability. If, on the contrary, the determinant is positive, then there are three eigenvalues with positive real value, i.e., the system is totally unstable.

\subsection{Proof of proposition 3.1}

Using 2.1 and 2.17, it can easily be shown that $u^{*}\left(=-g_{R}\right)>0$ ensures that the utility integral converges along a steady-state growth path; similarly, it is easy to show that $u^{*}>0$ guarantees the fulfilment of the transversality conditions 2.12 and 2.13. Hence, existence and the statements a) and b) follow directly from sections 2.1, 2.2 and 7.1. As to statement c), by 2.34 and appendix 7.1, $\gamma<1$ and 
stability (by statement a) stability is equivalent to saddle point stability) ensures $D>0$. Therefore, by 2.20 ,

$$
g_{c} \geq 0 \Rightarrow(\alpha+\beta+\gamma-1) n \geq \gamma \rho \Rightarrow(\alpha+\beta-1) n \geq \gamma \rho-\gamma n>0
$$

from 2.1; but this implies

$$
n \geq \gamma \rho /(\alpha+\beta+\gamma-1)>0 \wedge \alpha+\beta>1
$$

\subsection{Proof of proposition 3.2 (the case $n=0$ )}

In this case, from 2.20 it is seen that a steady state with $g_{c}=0$ is not possible, since $\rho>0$. Further, by $2.20, D<0$ is a necessary condition for a steady state with $g_{c}>0$. When $D<0$, by $2.24 u^{*}>0 \Leftrightarrow \alpha>1$. $\alpha>1$ will also ensure that $z^{*}>0$ (from 2.23). Furthermore, for $D<0$ and $\alpha>1$, by $2.22 x^{*}>0$ $\Leftrightarrow(1-\gamma) \rho>-D \delta$; hence, the conditions $\gamma<1$ and $\rho>-\frac{D \delta}{1-\gamma}$ are required. This proves the first part of the proposition. As to the second part, when $D<0$ and $\gamma<1$, the steady state is totally unstable as seen in section 7.1.

\subsection{Proof of proposition 3.3 (the case $\alpha=1$ )}

In this situation, $x^{*}=u^{*}=\frac{\beta}{\gamma} n$, from 2.22 and 2.24 , so that positive population growth is necessary and sufficient for positive values of these two variables. By 2.23 and $3.2, z^{*}=\varepsilon g_{c}+\delta+\rho$ so that $g_{c} \geq 0 \Rightarrow z^{*}>0$.

From $3.2, g_{c}>0$ if and only if

$$
\varepsilon>1 \wedge \gamma<\frac{\beta n}{\rho-n} \quad \text { or } \quad \varepsilon<1 \wedge \gamma>\frac{\beta n}{\rho-n} .
$$

From 2.34, necessary and sufficient conditions for saddle point stability (by proposition 3.1 equivalent to stability) are that either

$$
\varepsilon>1 \wedge \gamma<1 \quad \text { or } \quad \varepsilon<1 \wedge \gamma>1 \text {. }
$$

\subsection{Proof of proposition 3.4 (the case $\varepsilon=1$ )}

In this case, from 2.24, $u^{*}=\rho-n>0$ (from 2.1) and $D=1-\alpha$. 
Subcase $\alpha<1$. Here, $g_{c}>0 \Leftrightarrow \gamma<\frac{\alpha+\beta-1}{\rho-n} n$, by 2.20. Hence, $n>0$ is also required in this case. As $z^{*}=\frac{1}{\alpha}\left(g_{c}+\delta+\rho\right), g_{c}>0 \Rightarrow z^{*}>0$. By, 2.22, $x^{*}=\frac{1}{\alpha}[(1-\gamma) \rho+(\beta+\gamma-1) n+(1-\alpha) \delta]>\frac{1}{\alpha}[(1-\gamma) \rho+(\beta+\gamma-1) n-\alpha(\rho-$ $n)+(1-\alpha) \delta]=(1-\alpha) z^{*}>0$, by 2.23 .

A necessary and sufficient condition for saddle-point stability (by proposition 3.1 equivalent to stability) in this case is $\gamma<1$ (from 2.34).

Subcase $\alpha>1$. Here, $g_{c}>0 \Leftrightarrow \gamma>\frac{\alpha+\beta-1}{\rho-n} n$, by 2.20. We still have $z^{*}=\frac{1}{\alpha}\left(g_{c}+\right.$ $\delta+\rho)>0$ when $g_{c}>0$. As to $x^{*}$, from 2.22 follows $x^{*}>0 \Leftrightarrow \gamma<1+\frac{\beta n-(\alpha-1) \delta}{\rho-n}$.

Now, when $\alpha>1$, by 2.34, $\gamma>1$ is necessary and sufficient for saddlepoint stability, given that the steady state exists. But $\gamma>1$ is only consistent with the existence of the steady state, cf. the existence requirements $\alpha>1$ and $\gamma<1+\frac{\beta n-(\alpha-1) \delta}{\rho-n}$, if $n>0$. Hence, the conclusion that also $n>0$ is necessary for saddle point stability (hence stability).

Remark. Logically it would be sufficient to say that, in the case $\alpha>1$, a steady state is stable if and only if $\gamma>1$. We prefer, however, to put it the way it is done in the proposition, making the necessicity of $n>0$ explicit.

\section{References}

[1] Aghion, Philippe, \& Peter Howitt, 1998: Endogenous Growth Theory. MIT Press, Cambridge, Massachussetts.

Arrow, Kenneth J., 1962: The Economic Implications of Learning by Doing, Review of Economic Studies, vol. 29, pp. 155-173.

Barro, Robert J., \& Xavier Sala-i-Martin, 1995: Economic Growth. McGrawHill, New York.

Bond, Eric W., Ping Wang, and Chong K. Yip, 1996: A General Two-Sector Model of Endogenous Growth with Human and Physical Capital: Balanced Growth and Transitional Dynamics, Journal of Economic Theory, vol. 68, No. 1. 
Dasgupta, Partha, \& Geoffrey Heal, 1974: The Optimal Depletion of Exhaustible Resources, Review of Economic Studies, Symposium Issue, pp. 328.

Dasgupta, Partha, \& Geoffrey Heal, 1979: Economic Theory and Exhaustible Resources. Nisbet/Cambridge University Press.

Eicher, Theo S., \& Stephen J. Turnovsky, 1999: Non-Scale Models of Economic Growth, Economic Journal, vol. 109, pp. 394-415.

Groth, Christian, 1992: Endogen teknologisk udvikling (On a Model of Endogenous Growth). Nationaløkonomisk Tidsskrift, vol. 130, pp. 350-59.

Jones, Charles I., 1995: R \& D-Based Models of Economic Growth. Journal of Political Economy, vol. 103, pp. 759-784.

Lucas, Robert E., 1988: On the Mechanics of Economic Development. Journal of Monetary Economics, vol. 22, pp. 3-42.

Rebelo, Sergio, 1991: Long- Run Policy Analysis and Long-Run Growth. Journal of Political Economy, vol. 99, 3, pp. 500-521.

Robson, A. J., 1980: Costly Innovation and Natural Resources. International Economic Review, vol. 21, pp. 17-30.

Romer, Paul M., 1990: Endogenous Technological Change. Journal of Political Economy, vol. 98, S71-S102.

Scholz, Christian M., \& Georg Ziemes, 1996: Exhaustible Resources, Monopolistic Competition, and Endogenous Growth. Mimeo, University of Kiel.

Schou, Poul, 1999: Polluting Nonrenewable Resources and Growth. Forthcoming in Environmental and Resource Economics.

Solow, Robert M., 1994: Perspectives on Growth Theory. Journal of Economic Perspectives, vol. 8, pp. 45-54.

Stiglitz, Joseph, 1974: Growth with Exhaustible Natural Resources: Efficient and Optimal Growth Paths. Review of Economic Studies, vol. 41, Symposium Issue, pp. 123-137. 
Stokey, Nancy, 1998: Are There Limits to Growth? International Economic Review, vol. 39, pp. 1-31.

Takayama, Akira, 1980: Optimal Technical Progress with Exhaustible Resources. In: Kemp, M. C., \& N. Van Long (eds.): Exhaustible Resources, Optimality, and Trade. North-Holland, Amsterdam 1980. 